\title{
Realidad Virtual (HMD) e Interacción desde la Perspectiva de la Construcción Narrativa y la Comunicación: Propuesta Taxonómica
}

\section{Virtual reality (HMD) and Interaction from the Perspective of Narrative Construction and Communication: Taxonomic Proposal}

\author{
Jose Luis Rubio Tamayo \\ Doctor en Creatividad Aplicada \\ (Universidad Complutense de Madrid) \\ Manuel Gértrudix Barrio \\ Profesor Titular de Comunicación Digital \\ (Universidad Rey Juan Carlos)
}

Fecha de recepción: 14 de abril de 2016

Fecha de revisión: 10 de julio de 2016

Para citar este artículo: Rubio-Tamayo, J. L. y Gértrudix Barrio, M. (2016):

Realidad Virtual (HMD) e Interacción desde la Perspectiva de la Construcción Narrativa y la Comunicación: Propuesta Taxonómica, Icono 14, volumen 14 (2), pp. 1-24. doi: 10.7195/ri14.v24i2.965 


\section{Resumen}

La realidad virtual es un término popularizado por varios autores a partir de los años 80 y 90 para definir los rasgos de los entornos inmersivos generados de manera artificial por medios digitales. Desde las primeras tentativas de desarrollo de entornos sensorialmente inmersivos como Sensorama (1962), los primeros simuladores de vuelo de los 60 o el experimento Aspen Movie Map, la inmersión en entornos virtuales se ha transformado, dando lugar a experiencias que exploran nuevas posibilidades en ámbitos de la comunicación y la narrativa. El artículo realiza una revisión sistemática de la literatura científica de estos fenómenos, y del surgimiento de áreas interdisciplinares como el diseño de interacciones o la experiencia de usuario, para proyectar una clasificación taxonómica de los elementos que conforman la dimensión narrativa en el uso de la realidad virtual, generando modelos que complementen la configuración de los actuales. La propuesta taxonómica supone un paso cualitativo en el estudio de las dimensiones interactivas entre la tecnología, la persona, y el medio.

Palabras clave: Virtualidad - Simulación - Interactividad - Narrativa - Entornos virtuales - Inmersión - Taxonomía

\section{Abstract}

Virtual reality is a term popularised by some authors since the eighties and the nineties, in order to define features for immersive environments generated artificially by digital means. Immersive processes in virtual environments have dramatically changed since early experiences in this area, such as Sensorama (1962), flight simulators (in the sixties) or Aspen Movie Map Experiment (1978). Those changes offer new possibilities in communication and storytelling domains. This work does a systematic literature review about those phenomena, approaching also the emergence of interdisciplinary fields such as interaction design or user experience. The study aims also to configure a taxonomic classification of components in the domain of storytelling for the use of virtual reality technologies, trying to generate models which help to complement the current ones. This taxonomy is a qualitative milestone for studying interactive dimensions between technology, person and environment.

Key Words: Virtuality - Simulation - Interactivity - Storytelling - Virtual environments - Immersion - Taxonomy 


\section{Introducción}

La primera descripción conocida de un sistema de realidad virtual se puede encontrar en la obra de ciencia ficción de los años 30 Pygmalion Spectacles, del escritor Stanley Weinbaum (1935). En la obra, el protagonista conoce a un inventor que desarrolla un sistema inmersivo de visionado de películas y de simulaciones, donde no solamente se puede ver y oír el contenido digital, sino que también es posible interactuar de manera táctil u olfativa. El propio autor lo describe así:

Te encuentras dentro de la trama, hablas a las sombras (personajes) y ellas te responden, $\mathrm{y}$, en lugar de estar en una pantalla, la historia se encuentra toda alrededor tuyo". [You are in the story, you speak to the shadows (characters) and they reply, and instead of being on a screen, the story is all about you, and you are in it."]

Weinbaum (1935), en Pygmalion's Spectacles.

Ya en los años 60, Marshall McLuhan (1964) usa la frase "el medio es el mensaje" para reflejar la influencia determinante del primero en cómo este mensaje va a ser percibido e interpretado por el receptor. Es, a este respecto, el propio medio el que influye, además, en la configuración de la estructura narrativa de aquello que se pretende representar o comunicar, como bien indica McLuchan, y como bien reflejan este tipo de tecnologías, cuyos canales de comunicación han ido evolucionando. La realidad virtual -y el tipo de proyecciones tecnológicas con las que se encuentran relacionada- se conforma, así, como un potente medio de construcción narrativa y de comunicación cuyas posibilidades de aplicación todavía no están cuantificadas.

Es en esta misma década cuando surgen también las primeras tentativas de desarrollo de entornos sensorialmente inmersivos como Sensorama (1962), Telesphere Mask (Heilig, 1960), los primeros simuladores de vuelo de los 60 o el experimento Aspen Movie Map (en Naimark, 1978, en Marti, 2006), desarrollado por el MediaLab del MIT a finales de los años 70, y que es considerado como un precursor de Google Street View. Es también en esta década cuando surgen las primeras aproximaciones 
al diseño de dispositivos que posibilitan la interacción con la información generada de manera artificial. La primera tentativa de casco de realidad virtual (HMD o headmounted display) es desarrollada por Sutherland en esa década (en Sutherland, 1965, en McLellan, 1996). Debido a las limitaciones obvias de la época, el dispositivo era muy elemental para los estándares actuales. Sin embargo, Sutherland describe, en su publicación The ultimate display, el potencial del realismo inmersivo que imaginaba en un futuro uso de esta tecnología, como una metáfora de Alicia en el País de las Maravillas, y que no estaría exenta de riesgos. Sutherland imaginaba, así, también una computadora que podía ejercer una influencia y un control sobre la materia:

El ultimate display [el dispositivo inmersivo que él describía] sería una habitación en la cual la computadora puede controlar la existencia de la materia. Una silla dispuesta en dicha habitación sería suficiente para sentarse en ella. Unas esposas situadas en esta habitación tendrían el poder de confinar al usuario, y una bala podría ser fatal. Con una programación adecuada, tal dispositivo podría ser literalmente el País de las Maravillas en el que Alicia se movía.

Sutherland (1965, p.2)

El término realidad virtual comienza a popularizarse, no obstante, en los años 80, adquiriendo mayor relevancia a finales de la década y a principios de los 90 . Así, los mayores avances, tanto en el campo teórico y conceptual como en la práctica, encuentran un punto de inflexión en 1989 gracias a las aportaciones de Jaron Lanier (en Lanier y Biocca, 1992, en Steuer, 1992), en sus estudios de las aplicaciones e implicaciones de esta tecnología. Si bien fueron autores quienes como Lanier contribuyeron a popularizar el término a finales de los años 80 , el origen el mismo se remonta a los años 30, misma época en que fue escrita la obra de Weinbaum (1935), si bien es cierto que Weinbaum no empleó ese término. El término réalité virtuelle aparece por primera vez en la colección de manifiestos publicados por Artaud en 1938 (en Artaud, 1958), bajo el título de Le theatre et son double (El teatro y su doble). Krueger también emplea el término realidad artificial en los años 70 (en Krueger, 1983), que finalmente no llegó a popularizarse, si bien hacía referencia al mismo tipo tecnologías que permitían interactuar con la información artifi- 
cial. Broderick (1982), por su parte, también incluye el término realidad virtual en la trama ficticia de su novela The Judas Mandala. La novela de ciencia ficción Snow Crash, de Stephenson (1992), daría otro giro de gran relevancia en al incorporar el término metaverso. La inclusión de este término, posteriormente usado por la literatura científica, supondría un punto de inflexión cuantitativo relevante a la hora de definir los rasgos y la complejidad que pueden adquirir los entornos virtuales. Entre estos rasgos, se encontrarían, por ejemplo, la persistencia o capacidad de funcionamiento del sistema virtual con independencia del o los usuarios que haya conectados. También encontraríamos la capacidad que ofrece el metaverso a los usuarios conectados para interrelacionarse dentro del sistema, aunque a día de hoy estas posibilidades se encuentran en proceso de estudio.

Es también en los años 80 cuando emerge esta visibilidad del fenómeno y la literatura científica revela una vez más su interés por la proyección de este tipo de tecnologías. Asistimos, a partir de la popularización llevada a cabo por Lanier, a la emergencia de estudios y aproximaciones teóricas que llevaron a cabo tentativas de clasificación taxonómica (Rheingold, 1991, Lanier, 1992, Lanier y Biocca 1992). Estas tentativas proyectan definir el proceso de inmersión de una persona en un entorno artificial, creado mediante tecnologías digitales, argumentando que el nivel de inmersión difiere en función de la naturaleza de la interfaz. Es en las mismas fechas cuando Caudell y Mizell (1992) aportan estudios sobre la realidad aumentada, y Milgam y Kishino (1994), realizan una clasificación taxonómica que denominan continuo de la virtualidad.

Por otro lado, surgen, de manera paralela, áreas del conocimiento interdisciplinares, como el diseño de interacciones (ID) (Borchers, 2001, Sharp, 2003, Kaptelinin \& Nardi, 2006, Zimmermann et al., 2007, Dix, 2009, Sedig \& Parsons, 2013, Tu et al., 2016), la experiencia de usuario (UX) (Hassenzahl, M., \& Tractinsky, 2006, Garrett, 2010, Albert \& Tullis, 2013, Karapanos, 2013, Nogueira et al., 2013, Backholm \& Alisawi, 2016), la interacción humano-computadora (HCI) (Rekimoto y Nagao, 1995, Smith-Atakan, 2006, Schneiderman, 2016), o la computación afectiva (AC) (Picard, 1995, Tao \& Tan, 2005, Wu et al, 2015). Estas analizan, desde perspectivas heterogéneas, las relaciones entre el/la usuario/a, la tecnología, las interfaces y el entorno, realizando aportaciones relevantes al ámbito de las ciencias de la información y la comunicación. 


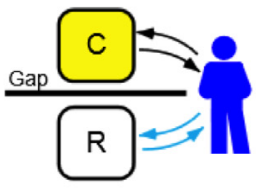

(a) GUI

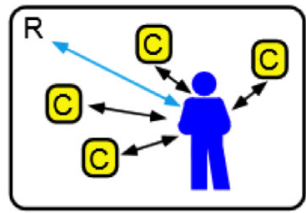

(c) Ubiquitous Computers

C. Computer World

R Real World

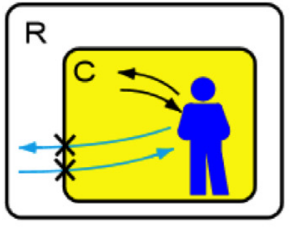

(b) Virtual Reality

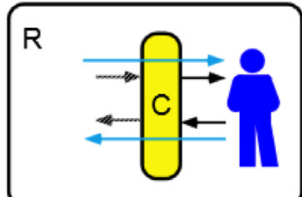

(d) Augmented Interaction

$\leftrightarrow$ Human - Computer Interaction $\leftrightarrow$ Human - Real World Interaction

- Real World - Computer Interaction

Figura 1: Taxonomía de los niveles de interacción HCI y HRWI (human-real world interaction, o interacción persona-mundo real) (Rekimoto y Nagao, 1995), donde se observa la naturaleza del espacio con el que interactúan las/los usuarias/os.

[Traducción: Gap: Brecha / GUI: Interfaz gráfica de usuario / Virtual Reality: Realidad Virtual/ Ubiquitous Computers: Ordenadores Ubicuos /Augmented Interaction: Interacción Aumentada/ Computer World: Mundo Virtual / Real World: Mundo Real / Human - Computer Interaction: Interacción Humano - Computadora / Human - Real World Interaction: Interacción humano

- Mundo real / Real world - Computer Interaction: Interacción mundo real - Computadora]

\section{Método}

La investigación se fundamenta en una exhaustiva revisión de la literatura científica dentro del área de estudio relacionada con las aplicaciones de la realidad virtual. Partiendo de los primeros estudios de Lanier y Biocca (1992), se han examinado un total de 85 referencias que abarcan los ámbitos de la realidad virtual, el continuo de la virtualidad, las aproximaciones a la interacción, o la dimensión comunicativa (Ryan, 2001, Sherman y Craig, 2002, Churchill et al., 2012). En el análisis de las aportaciones seleccionadas se ha llevado a cabo un rastreo teórico crítico y evaluativo con la finalidad de hallar un enfoque integrador que aporte 
una nueva perspectiva sobre la aplicación de la Realidad Virtual (HMD) y la Interacción desde la perspectiva de la construcción narrativa y la comunicación.

Se han analizado también artículos de referencia recientes que ponen de relieve las proyecciones y limitaciones tecnológicas actuales. También se realiza una revisión de artículos de investigación, publicados en los últimos cinco años, que analizan las aplicaciones más recientes de la realidad virtual en diversos campos, y sus avances en el contexto de la interactividad.

Este espacio actualiza la necesidad de una elaboración teórica renovada que, a partir de tal revisión de la literatura científica y especializada, plantea poner de relieve la proyección actual de la tecnología en base a factores como la inmersión, la interacción o la simulación. Estos factores han sido configurados en base a un análisis de sus rasgos y características en literatura científica y temática reciente, a partir del uso de conceptos e ideas relacionados con la relación entre las personas, los dispositivos, y el entorno.

Para completar el estudio, se realiza una serie de contribuciones a la clasificación taxonómica propuesta por Milgram y Kishino (1994), en torno al continuo de la virtualidad, para finalizar enumerando algunas experiencias innovadoras recientes en esta área (Dai et al., 2016, Hsu, 2016), y su relación con factores cognitivos o ergonómicos (Aromaa \& Väänänen, 2016, Lackey et al., 2016). Terminamos con una propuesta (abierta) de clasificación taxonómica que ayude a una mejor comprensión del contexto en el que se sitúan los componentes en su relación con los diferentes niveles de realidad-virtualidad, desde el punto de vista de la narrativa o la interacción. Se plantea reconstruir, de manera esquemática, el estado del arte a partir de investigaciones precedentes, y con la intención de ayudar a investigaciones posteriores.

Estas aportaciones se contrastan mediante el examen de un conjunto de aplicaciones recientes que hacen uso de la realidad virtual, tratando de generar modelos que ayuden a complementar los actuales, y a realizar una clasificación taxonómica de los elementos que pasan a formar parte de la narrativa en el uso de la realidad virtual. 


\section{INNOVACIÓN TEÓRICA}

\section{Desarrollo}

\subsection{Revisión de la Literatura Científica en Realidad Virtual: Las perspectivas del medio}

A partir de la ya mencionada obra de Lanier y Biocca (1992), los estudios en el campo teórico de la realidad virtual como medio de comunicación se suceden en los 90 del siglo XX y en las dos primeras décadas del siglo XXI. Así, en los 90 destacan los estudios de Steuer (1992), definiendo la realidad virtual como un canal de información y un medio de comunicación en detrimento de la perspectiva exclusiva del dispositivo. También destacan los estudios de Cruz-Neira et al. (1993), en torno al propio desarrollo de la CAVE (Cave Assisted Virtual Environment), conformando un sistema de realidad virtual popularizado en los años 90, y que fue precursora de las experiencias de mapping en fachadas en las últimas décadas (Cardona, 2015).

Las propuestas de aplicación de la realidad virtual en las dos primeras décadas del siglo XXI son observables en un amplio rango de disciplinas. Su potencial como herramienta de comunicación y de transmisión de la información es explorado en áreas tan dispares como la neurociencia (Riva et al., 2004, Rizzo et al. 2004, Levin et al. 2015), el turismo y el patrimonio cultural (Guttenlag, 2010. Mendoza et al., 2015, Ortega et al., 2015), la conducción de vehículos y simulación de uso de máquinas (0ng \& Nee, 2013, Tudor et al., 2015). Otros autores exploran el potencial de la realidad inmersiva para potenciar el desarrollo de entornos virtuales colaborativos. Seidel y Chatelier (2013) plantean esta reflexión en torno a la idea conceptual de un entorno colaborativo inmersivo para ser aplicado en áreas como el aprendizaje. Revelan, igualmente, las dificultades que plantea el desarrollo de una arquitectura conceptual de las interacciones en un contexto de aprendizaje ( $p$. 10). Así, frente a la proyección idealizada del propio medio, plantea los obstáculos que surgen en experiencias de implementación. Así, confrontan la perspectiva idealizada del realismo situacional (vivir experiencias sensoriales con ahorro de tiempo y dinero) y la idea de herramientas y contextos adaptadas al usuario con el énfasis que se hace en ciertas competencias y el exceso de instruccionismo en el que a veces se cae. 
Churchill et al. (2012, p. 9), desde la perspectiva de la realidad virtual como potencial medio de comunicación y de colaboración, también describen parte de los rasgos que precisan los entornos de realidad virtual para ser colaborativos. Entre estos rasgos que precisan, se encuentran:

- Que el entorno sea un espacio colectivo.

- Que permita adquirir conciencia de la existencia de otros

- Que permita dinámicas de negociación y comunicación fluidas.

- Que presente múltiples puntos de vista.

Ya en los primeros años del siglo XXI, Ryan (2001) propone que la propia narrativa se conforma como un sistema de realidad virtual. Ryan plantea fundamentalmente la idea de la realidad virtual como una metáfora del arte total, planteando así el interrogante sobre las posibilidades de representación que ofrece esta tecnología. La autora reivindica, de este modo, una cultura más implicada con el fenómeno de la interactividad.

Sherman y Craig (2002) integran también en sus trabajos los estudios sobre la relación entre realidad virtual, comunicación, interacción, interfaces 0 diseño. En lo referente al desarrollo de sistemas, los trabajos de Reiners et al. (2015) ponen el foco en el desarrollo de un prototipo de simulador onmidireccional (con seis grados de libertad) estéreo fotorrealista. Por su parte, en la búsqueda de parámetros para la mejora de la usabilidad, Guerrero et al. (2015) investigan en torno al desarrollo de un prototipo simplificado de simulación de la realidad virtual. Y, en una perspectiva macro, el potencial de comunicación y los retos a los que se enfrenta esta tecnología es explorada por Biocca y Levy (2013).

Las aplicaciones del estudio de la interactividad a la realidad virtual se ponen de relieve en investigaciones recientes relacionadas con las ciencias de la salud. Estas van desde la simulación para el entrenamiento y la práctica de los 


\section{INNOVACIÓN TEÓRICA}

profesionales de la medicina, hasta el tratamiento y recuperación de pacientes. Entre estos estudios encontramos las publicaciones de Sharar et al. (2014), que analizan la aplicación de la realidad virtual a pacientes aquejados de quemaduras de gravedad, proporcionando, de este modo, al paciente, un entorno de distracción inmersivo. En una línea parecida trabajan Hoffman et al. (2014), desarrollando un sistema de realidad virtual con 0culus Rift para terapia ocupacional en unidades de quemados en hospitales infantiles. Gorini y Riva (2014) también investigan en la aplicación de las posibilidades de interacción de realidad virtual en trastornos de ansiedad. Por su parte, autores como Meshulam et al. (2016) investigan en torno al potencial de las interacciones simples para revelar rasgos complejos de la actividad neuronal. Kandalaft et al. (2013) investigan en torno al potencial de la tecnología de realidad virtual para el entrenamiento cognitivo de adultos con un grado de autismo elevado.

Las aplicaciones de los estudios de la interacción con la tecnología de realidad virtual encuentran exponentes de interés en el campo de la educación. Ya en los primeros albores de la realidad virtual encontramos trabajos que proyectan en uso de esta tecnología para el desarrollo de entornos de aprendizaje inmersivos (Pstoka, 1995). Kaufmann et al. (2000) desarrollan, a principios del siglo XXI, uno de los primeros entornos en realidad virtual para el aprendizaje de las matemáticas y la geometría, llamado Construct 3D. En la presente década, Fogarty el al. (2015), ponen el foco en la complejidad de la realidad virtual tridimensional para desarrollar estrategias de implementación eficientes en el contexto educativo. Thorsteinsson (2013) pone de relieve, también, el potencial de este conjunto de tecnologías y de los estudios de interacción para proyectar los siguientes pasos de innovación en la educación. Y en ámbitos específicos de esta área, encontramos ejemplos de aplicaciones de gran interés. Así, Bissonnette et al. (2016) realizan estudios de aplicación de esta tecnología para la mejora en el rendimiento de la interpretación musical. Por su parte, Moreno Toledano y Mogel Villalba (2015) se aproximan, en sus investigaciones, al potencial de representación narrativo y retórico en las nuevas culturas mediáticas en relación a los mundos virtuales. También podemos encontrar investigaciones de gran originalidad, que plantean incluso la perspectiva de género, desde el punto de vista del feminismo, (D'Ignazio et al., 2016), contribuyendo a la apertura de líneas de 
Realidad Virtual (HMD) e Interacción desde la Perspectiva de la Construcción... | 11

INNOVACIÓN TEÓRICA

investigación conceptuales para profundizar en los usos de esta tecnología para la optimización de los procesos de comunicación.

\subsection{La propuesta de modelo taxonómico para estado del arte}

La realidad virtual se proyecta como un sistema de interacción con diferentes niveles de inmersión, y que abarca varias dimensiones sensoriales. A partir de los estudios de las disciplinas a las que ya se ha hecho referencia (HCI, ID, UX), el diseño de sistemas de realidad virtual tiene que tener en cuenta numerosos factores, que abarcan desde el cognitivo, el ergonómico, el diseño, las posibilidades y las limitaciones de la tecnología en el contexto actual. A esto se suma el estudio de las teorías de la comunicación y su implementación en los sistemas tecnológicos emergentes, entre ellos los entornos inmersivos de realidad virtual. Además, tenemos referentes anteriores como el cine o la literatura, que son definidos como sistemas de realidad virtual conceptuales en algunos estudios (Ryan, 2001). Estos, debido a su naturaleza, entrarían en una clasificación taxonómica que ya fue definida por Milgram y Kishino (1994), y a partir de la cual se proyecta una relación directa con las dimensiones relativas a la percepción humana, la interacción con el medio o la cognición, así como la construcción de historias.

A partir de las observaciones en la literatura científica y en las aplicaciones del contexto actual, se plantea la configuración de una estructura que sitúa los diferentes componentes dentro del continuo de la virtualidad. En este planteamiento se tienen, también, a su vez, en cuenta, áreas de estudio interdiciplinares que abarcan los diferentes niveles de realidad-virtualidad, y que se estructuran en torno a las diferentes dinámicas de comunicación e interacción.

En la Figura 2 observamos la relación que tienen los diferentes niveles de interacción y cognitivos con la clasificación taxonómica llevada a cabo originariamente por Milgram y Kishino (1994). 


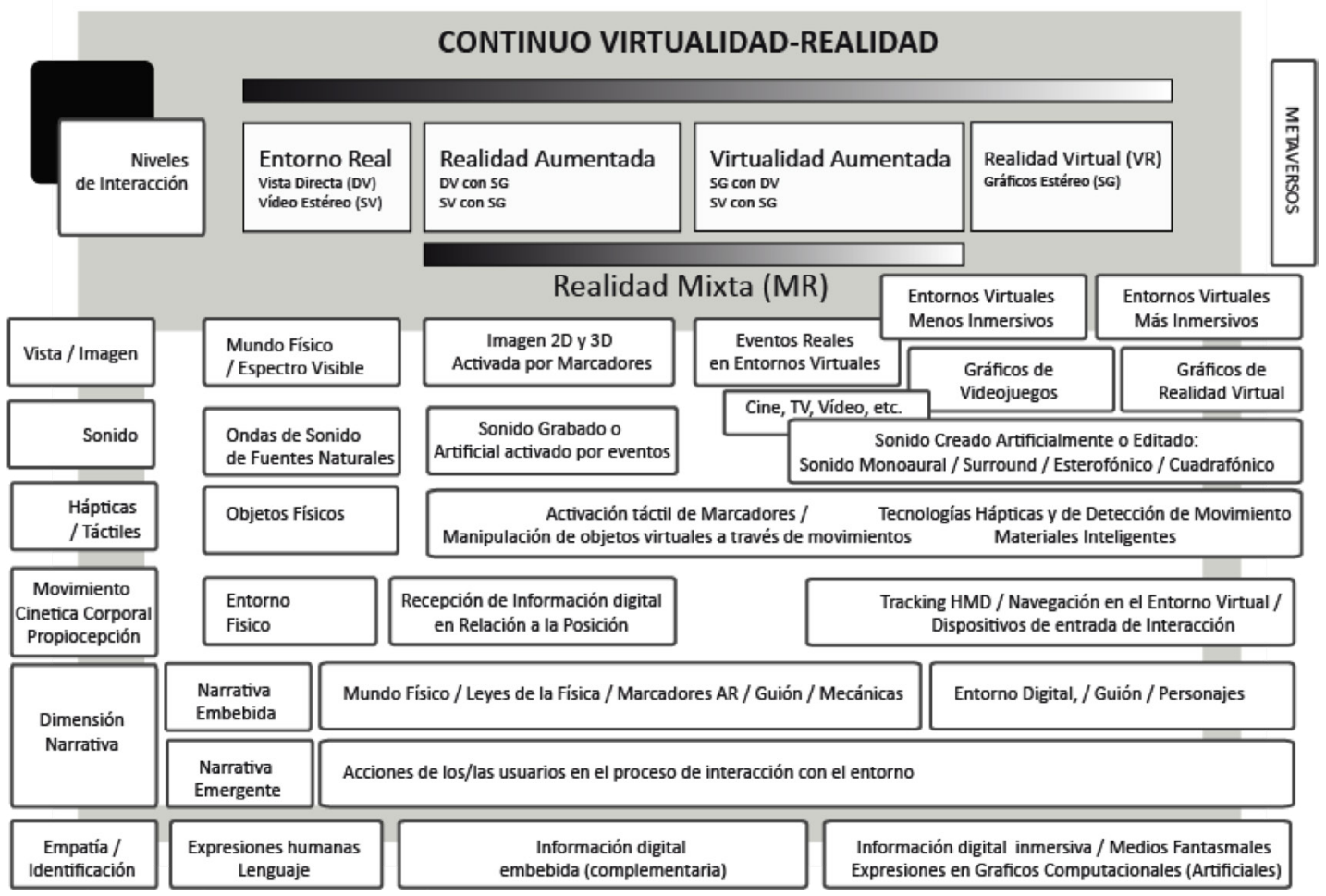

Figura 2: Clasificación taxonómica de los niveles de recepción de información en el continuo de la virtualidad.

En las aplicaciones en el ámbito de la comunicación, el estudio de la narrativa juega un papel esencial. A la hora de configurar un sistema donde se producen interacciones con un entorno de realidad virtual, podemos distinguir entre dos tipos de narrativa. Por un lado, encontraríamos la narrativa embebida, que es aquella que, como explica Monchán (en Rubio-Tamayo, 2015) la que se encuentra dentro del propio sistema. Es la que corresponde, de manera implícita, al entorno, y a los elementos que no se pueden desligar de las mecánicas del mismo. En el otro lado, encontramos la narrativa emergente, que es aquella en un sistema interactivo a medida que el/la usuario/a interactúa con el propio sistema. En la figura 3 podemos observar el planteamiento de la relación entre las dos propuestas narrativas y la clasificación taxonómica realizada por Milgram y Kishino (1994) del continuo de la virtualidad. 


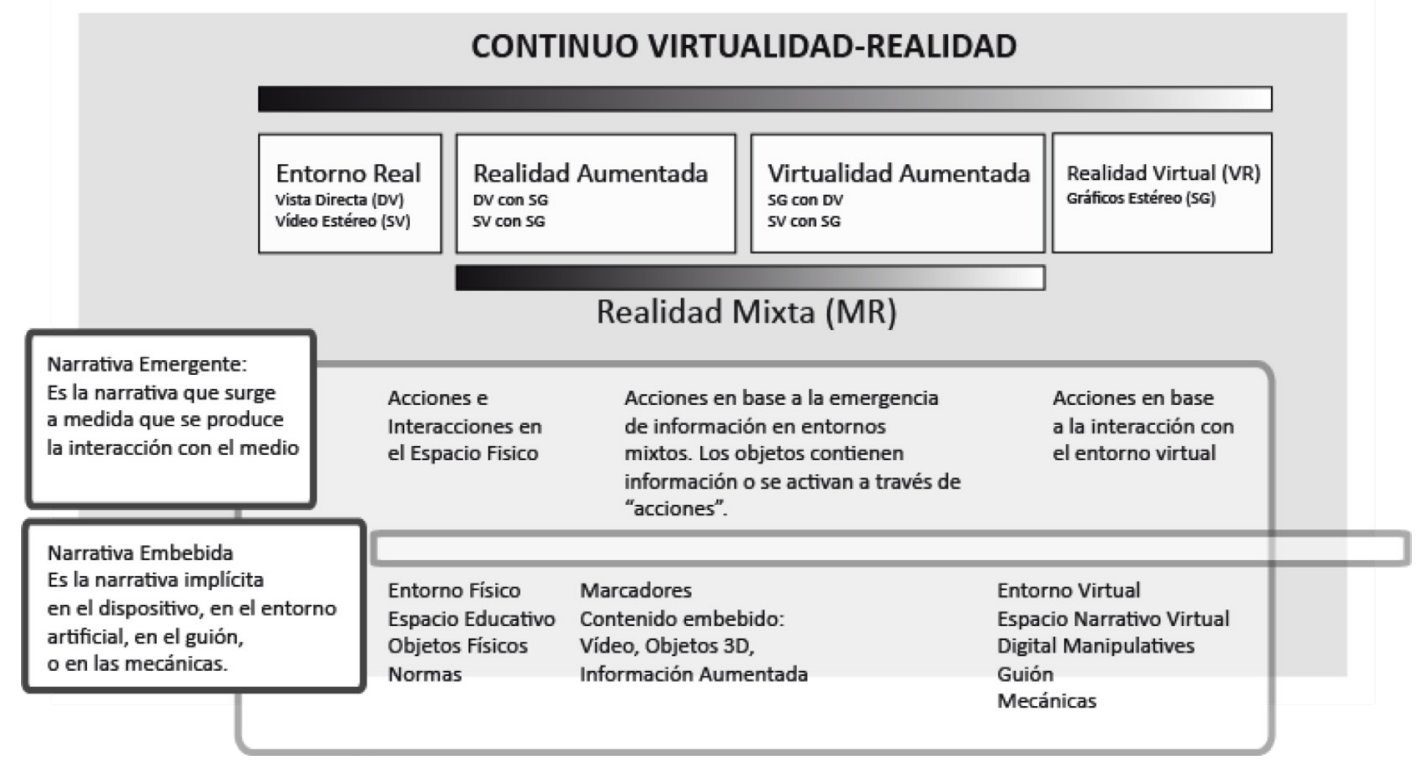

Figura 3: La narrativa emergente y la narrativa embebida en el continuo de la virtualidad. Elaboración propia (2015).

\section{Conclusiones}

\section{Aportaciones teóricas y contexto}

En el contexto actual, varios estudios observan la realidad virtual como un medio de comunicación inmersivo, con diferentes grados en su potencial de interacción. En esta área, Lorenz et al. (2016) exploran el desarrollo de dos métodos de navegación en entornos de realidad virtual para simular el proceso de andar. Otros autores como Aromaa y Väänänen, K. (2016) ponen de relieve la relación entre la realidad virtual, el proceso de prototipado, el diseño y los factores ergonómicos, tratando de desarrollar estándares para implementar prototipos virtuales en el proceso de desarrollo de producto. Dai (2016) trabaja en la misma línea, realizando investigaciones en torno a la implementación de técnicas de realidad virtual para su uso como herramienta de diseño de prototipos. Los avances en los últimos años también se hacen presentes en la aplicación de la interfaz cerebro-computadora para la mejora de los procesos de interacción-comunicación en el uso de la realidad virtual. Así, Hsu (2016) desarrolla un modelo de interfaz cerebro-computadora (BCI) para aplicaciones en telemedicina. 


\section{INNOVACIÓN TEÓRICA}

Actualmente, la emergencia de aplicaciones de esta tecnología se fundamenta en las necesidades de desarrollo de nuevos procesos y medios de comunicación. Estos nuevos horizontes de comunicación que plantea una tecnología que posibilita una inmersión directa se incrementan exponencialmente. Un ejemplo relevante es el caso del uso de la realidad virtual por Médicos sin Fronteras para situar al espectador en un campo de refugiados, en un proyecto llamado \#Seguirconvida. Una cámara de 360 grados filma un campo de refugiados, introduciendo al usuario en la realidad del día a día de estas personas. La intención de estas experiencias es, sin duda, fomentar la empatía a través de una inmersión. Esta línea argumental también se esgrime en al ámbito de la aplicación de la realidad virtual a los videojuegos. Incluso en el contexto de la ficción, la realidad virtual inmersiva plantea problemáticas que hasta ahora no había planteado el videojuego en una pantalla. Tal y como explica Santo (2016), existe una diferencia fundamental entre apretar un botón de un mando para disparar a un personaje en un videojuego, con la distancia de una pantalla, a hacerlo con una pistola y unas gafas de realidad virtual (aunque sea un acto de ficción). Esta afirmación la realiza en referencia a un mod de GTA V desarrollado por Joseph Delgado para realidad virtual (en Santo, 2016). No existe todavía un relevante número de estudios comparativos sobre este incremento de grado de empatía con los elementos que componen el mundo inmersivo en la realidad virtual, pero muchas de las aplicaciones llevan implícito el incremento en el factor empático. En este aspecto, también incide el experimento The Machine to be Another (La Máquina para ser 0tro), desarrollado por BeAnotherLab. Este experimento sitúa a dos personas en la piel de la otra. Mediante el uso de dispositivos de realidad virtual (HMD) y una cámara, se coordinan los movimientos para adquirir, de manera inmersiva, otra dimensión corporal.

El uso de la realidad virtual también pone de relieve su uso potencial como herramienta para la transformación de la realidad social. La implementación en contextos educativos, ayuda a investigar en metodologías de aprendizaje que fomentan la colaboración grupal, mediante el aprovechamiento del espacio del aula. Este es el caso del proyecto Espacio, Inmersión, Interacción, llevado a cabo por ArsGames en colaboración con LABoral, donde la realidad virtual se articula como una herramienta participativa, a través de la proyección de aquello que observa el usuario en las HMD, en el espacio común. Así, se aprovecha el potencial de motivación que tiene, por un lado, la propia realidad virtual, y por otro se construye una comunidad colaborativa, en la que los objetos de referencia en el espacio 
físico (libros, fotografías), también forman parte de esa narrativa emergente que va surgiendo en el proceso de interacción con la tecnología. A través de la inmersión de un usuario en un entorno de realidad virtual con una narrativa embebida elemental, y su proyección en una pizarra digital o un proyector, el entorno virtual se transforma en un entorno compartido, dando lugar a dinámicas que aprovechan también la interacción con el propio espacio físico. Así, este escenario de innovación en torno a la interacción con el espacio, presenta un potencial de gran interés para las líneas de investigación en torno a la implementación de metodologías de aprendizaje colaborativas. La implementación de nuevos dispositivos controladores, tales como Kinect o Leap Motion, contribuyen, además, a dinamizar el proceso de interacción humano-computadora, lo que contribuye, a su vez, a integrar nuevas dimensiones en la interacción entre la persona y el entorno físico.

Lo que sin duda ocurrirá, es que la realidad virtual va a continuar evolucionando en la configuración de su propio lenguaje narrativo y sus propias dinámicas de interacción, a medida que la tecnología vaya permitiendo un mejor rendimiento de la herramienta.

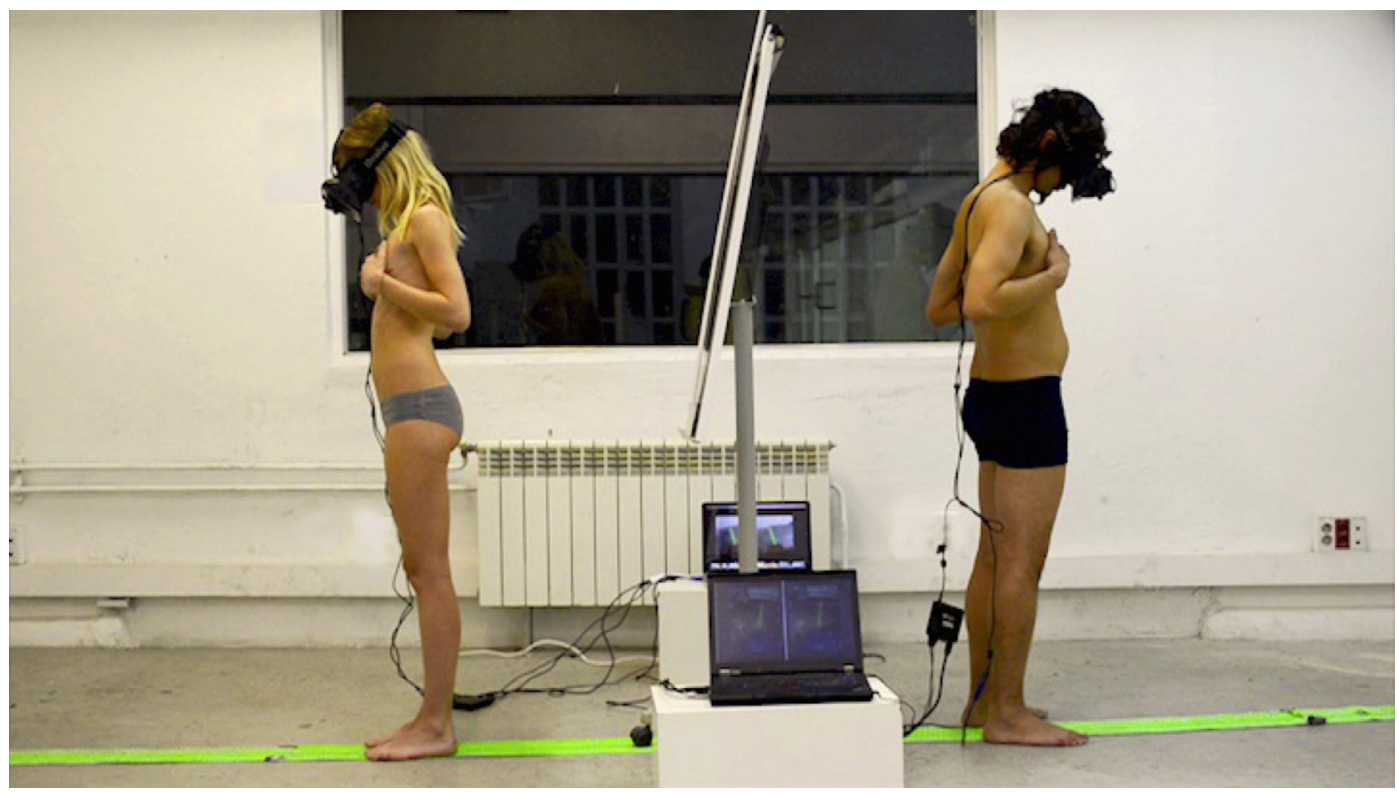

Figura 4: GenderSwap, desarrollado por BeAnotherLab. Imagen recuperada en 2015-12-05 de http://beanotherlab.org/gender-swap-experiment/ 
Podemos concluir que, a partir del análisis del conjunto de experiencias a las que se hace referencia en este trabajo, el proceso de evolución de la realidad virtual integra varias perspectivas, interrelacionadas entre sí:

- La perspectiva tecnicista: La que se basa en la mejora de los dispositivos desde un punto de vista exclusivo del rendimiento (gráficos, fotogramas por segundo, compatibilidad con tarjetas gráficas de equipos). Esta perspectiva plantea que las limitaciones tecnológicas son una cuestión estrictamente temporal, que se resolvería con la siguiente generación de dispositivos.

- La perspectiva conceptual: Esta perspectiva se fundamenta en la idea de lo que entendemos como realidad virtual, y qué esperamos de este tipo de tecnología.

- La perspectiva de la comunicación-interfaz-narrativa: Es la forma en que entendemos nuestra relación con los entornos virtuales, y su estudio desde las ciencias de la información y de la comunicación. Incluye, también, áreas de estudio interdisciplinares como la experiencia de usuario, la interfaz de usuario, o la interacción humano-computadora.

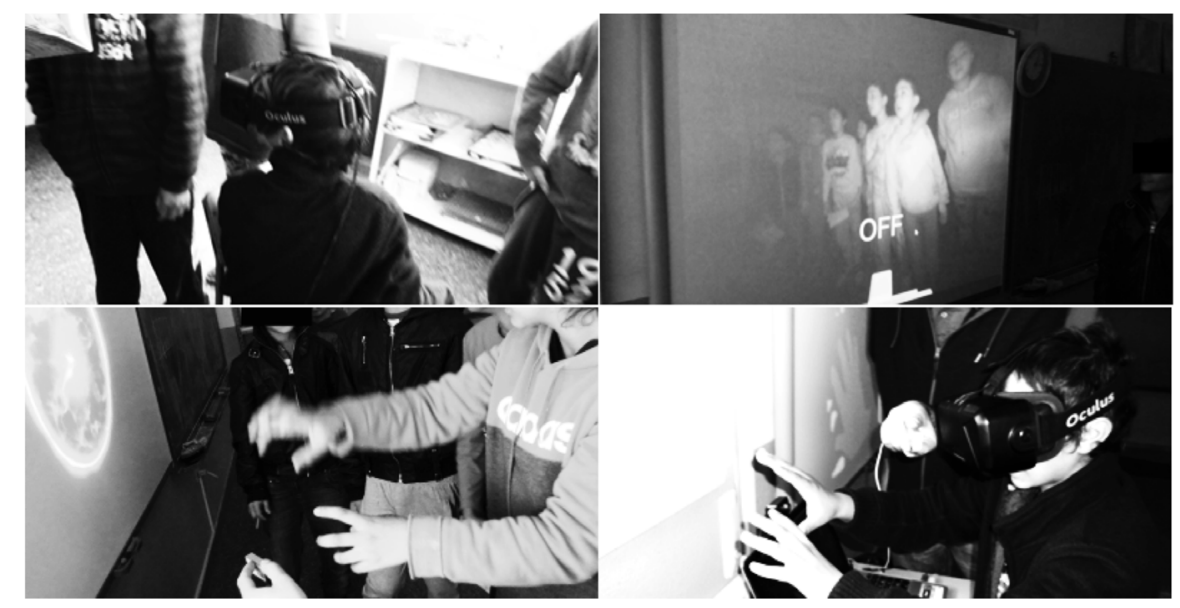

Figura 5: Sesiones del proyecto piloto Espacio, Inmersión, Interacción, que plantea experiencias en la escuela con el uso de realidad virtual y dispositivos infrarrojos, explorando la narrativa emergente en aula. ArsGames y LABoral. Fotos: Javier Silverio y Jose Luis Rubio-Tamayo (2015) 


\section{Discusión, perspectiva, y proyección}

Las perspectivas, a corto y medio plazo, de desarrollo de experiencias inmersivas, como ya hemos visto, dependen en gran parte de estos tres planteamientos relacionados con la realidad virtual: tecnológico, conceptual, y comunicación-interfaz-narrativa. Otros factores son también importantes a la hora de plantear escenarios en los que la realidad virtual evolucione conceptual, tecnológica y narrativamente. Entre estos, estaría el componente lúdico, cuyo estudio incrementa las posibilidades para la apertura de líneas de investigación de los componentes a lo largo del continuo realidad-virtualidad. Así, los juegos de realidad alternativa o ARGs, que utilizan en parte el espacio físico, ofrecen numerosas posibilidades en el estudio de la interacción con el espacio y la narrativa. Esto no hace sino incrementar el espectro de posibilidades para aplicaciones en las áreas que ya se han descrito en el presente estudio.

Es necesario también plantear la inclusión de nuevos conceptos y figuras retóricas asociados a este tipo de tecnología, y que faciliten la comprensión del lenguaje emergente que nace de la mano de la interacción y la comunicación con los entornos virtuales. Autores como Gértrudix, García y Gértrudix (2016) incorporan la metáfora del zoco digital, como lugar de intercambio de experiencias. Harrell (2013) incorpora el fenómeno denominado medios fantasmales (phantasmal media) al ámbito de la comunicación en la cultura digital. Estos medios fantasmales son definidos por Harrell como una combinación de imaginación sensorial y constructos culturales, y se componen de fenómenos cognitivos que incluyen la propia identidad personal, las metáforas, la narrativa o las categorías sociales. Estas aproximaciones, entre otras, suponen aportaciones de gran relevancia en lo referente a la construcción de una cultura proyectada en los medios digitales.

Las posibilidades de comunicación y de configuración de narrativa a través de los entornos virtuales se encuentran, a este respecto, sometidas a procesos de intervención por parte de usuarios. Así, se proyectan, en parte, hacia los aspectos que ya apuntaba Stephenson (1992) cuando se definían los rasgos de los metaversos, como la persistencia o la interacción entre los usuarios de ese espacio. En cierto modo, el continuo de la virtualidad es una proyección de la realidad física, y una 


\section{INNOVACIÓN TEÓRICA}

prolongación de la misma hacia otra concepción del espacio diferente, pero que hunde sus raíces en el espacio físico. A través de la construcción de estas metáforas y referencias, y de una cultura digital, la realidad virtual evoluciona como canal de comunicación con rasgos propios. A su vez, este espacio de realidad virtual puede ser abordado como un lugar de existencia teórica, que puede ser un constructo en el imaginario en lo que Bourdieu (1993) denomina espacio de posibles. Este espacio de posibles se manifestaría, en el campo de la realidad virtual, como el conjunto de aquellas aplicaciones y proyecciones teóricas que se encuentran en tensión con las limitaciones tecnológicas o estructurales de un contexto específico.

Para concluir, se observa una tendencia en la que cada área del conocimiento proyecta profundizar en unas necesidades de comunicación específicas. Esto implica adaptar sus contenidos y sus dinámicas de comunicación a sus propias necesidades, lo que pone de relieve la necesidad de continuar profundizando en los estudios de comunicación. Además, la relación entre la persona, la tecnología y el medio, proyecta, cada vez, un escenario de mayor complejidad en el ámbito de las ciencias de la información y la comunicación.

\section{Bibliografía}

Albert, W., \& Tullis, T. (2013). Measuring the user experience: collecting, analyzing, and presenting usability metrics. Newnes.

Aromaa, S., \& Väänänen, K. (2016). Suitability of virtual prototypes to support human factors/ergonomics evaluation during the design. Applied Ergonomics, 56, 11-18.

Artaud, A. (1958). The theater and its double (Vol. 127). Grove Press.

Backholm, A., \& Alisawi, R. (2016). U.S. Patent No. 20,160,057,651. Washington, DC: U.S. Patent and Trademark Office.

Biocca, F., \& Levy, M. R. (Eds.). (2013). Communication in the age of virtual reality. Routledge.

Bissonnette, J., Dubé, F., Provencher, M. D., \& Sala, M. T. M. (2016). Evolution of music performance anxiety and quality of performance during virtual reality exposure training. Virtual Reality, 20(1), 71-81.

Borchers, J. 0. (2001). A pattern approach to interaction design. Ai \& Society, 15(4), 359-376. 
Bourdieu, P. (1993). The Field of Cultural Production. History\&Criticism271

Broderick, D. (1982). The Judas Mandala

Caudell, T. P., \& Mizell, D. W. (1992, January). Augmented reality: An application of heads-up display technology to manual manufacturing processes. In System Sciences, 1992. Proceedings of the Twenty-Fifth Hawaii International Conference on (Vol. 2, pp. 659-669). IEEE.

Cardona, C. A. B. (2015). 3D Beaker: Video Mapping Tridimensional sobre Superficies Curvas.

Churchill, E. F., Snowdon, D. N., \& Munro, A. J. (Eds.). (2012). Collaborative virtual environments: digital places and spaces for interaction. Springer Science \& Business Media.

Cruz-Neira, C., Sandin, D. J., \& DeFanti, T. A. (1993, September). Surround-screen projection-based virtual reality: the design and implementation of the CAVE. In Proceedings of the 20th annual conference on Computer graphics and interactive techniques (pp. 135-142). ACM.

Dai, F., Felger, W., \& Göbel, M. (2016). Applying Virtual Reality to Electronic Prototyping-Concept. Virtual Prototyping: Virtual environments and the product design process, 326.

D'Ignazio, C., Hope, A., Michelson, B., Churchill, R., \& Zuckerman, E. (2016). A Feminist HCI Approach to Designing Postpartum Technologies:" When I first saw a breast pump I was wondering if it was a joke.".

Dix, A. (2009). Human-computer interaction (pp. 1327-1331). Springer US.

Earnshaw, R. A. (Ed.). (2014). Virtual reality systems. Academic press.

Fogarty, J., El-Tawil, S., \& McCormick, J. (2015, April). Exploring Structural Behavior and Component Detailing in Virtual Reality. In Structures Congress 2015 (pp. 2557-2564). ASCE.

Fraser, M., Glover, T., Vaghi, I., Benford, S., Greenhalgh, C., Hindmarsh, J., \& Heath, C. (2000, September). Revealing the realities of collaborative virtual reality. In Proceedings of the third international conference on Collaborative virtual environments (pp. 29-37). ACM.

Garrett, J. J. (2010). Elements of user experience, the: user-centered design for the web and beyond. Pearson Education. 


\section{INNOVACIÓN TEÓRICA}

Gértrudix, M., García, F., Gértrudix, F. (2016). El Zoco de Aprendizaje Digital: Una proyección sobre las competencias educativas de los jóvenes. En IV Congreso Internacional Ciudades Creativas, Madrid, 2016 (412-422)

González Áspera, A., Chávez Hernández, G. (2011).La realidad virtual inmersiva en ambientes inteligentes de aprendizaje. Un caso en la educación superior. Icono 14 (122-137)

Gonzalez-Franco, M., Hall, M., Hansen, D., Jones, K., Hannah, P., \& Bermell-Garcia, P. (2015, March). Framework for remote collaborative interaction in virtual environments based on proximity. In 3D User Interfaces (3DUI), 2015 IEEE Symposium on (pp. 153-154). IEEE.

Gorini, A., \& Riva, G. (2014). Virtual reality in anxiety disorders: the past and the future. Expert Review of Neurotherapeutics.

Guerrero, C. D. Q., Ballen, E. L. S., \& Manrique, W. J. S. (2015). Diseño de un prototipo de sistema de realidad virtual inmersivo simplificado. Ciencia $e$ Ingeniería Neogranadina, 18(1), 35-50.

Guo (2016). En desarrollo un Mod de Joseph Delgado para GTA V que le da soporte a las gafas de realidad virtual. En La Nación Arcadia [En línea]. 22 de febrero de 2016. Recuperado en 2016-03-30 <http://www.nacionarcadia.com/endesarrollo-un-mod-de-joseph-delgado-para-gta-v-que-le-da-soporte-a-lasgafas-de-realidad-virtual/>

Guttentag, D. A. (2010). Virtual reality: Applications and implications for tourism. Tourism Management, 31(5), 637-651.

Harrell, F. (2013). Phantasmal Media. An Approach to Imagination, Computation, and Expression. MIT Press.

Hassenzahl, M., \& Tractinsky, N. (2006). User experience-a research agenda. Behaviour \& information technology, 25(2), 91-97.

He, P. (2016). Virtual Reality for Budget Smartphones. Young Scientists Journal, (18), 50.

Hoffman, H. G., Meyer III, W. J., Ramirez, M., Roberts, L., Seibel, E. J., Atzori, B., ... \& Patterson, D. R. (2014). Feasibility of articulated arm mounted 0culus Rift Virtual Reality goggles for adjunctive pain control during occupational therapy in pediatric burn patients. Cyberpsychology, Behavior, and Social Networking, 17(6), 397-401. 
Heilig, M. L. (1960, 4 de octubre). Telesphere Mask [Patente] Recuperado en 201603-15. <http://www.mortonheilig.com/TelesphereMask.pdf>

Hsu, W. Y. (2016). Brain-computer interface connected to telemedicine and telecommunication in virtual reality applications. Telematics and Informatics. Kandalaft, M. R., Didehbani, N., Krawczyk, D. C., Allen, T. T., \& Chapman, S. B. (2013). Virtual reality social cognition training for young adults with high-functioning autism. Journal of autism and developmental disorders, 43(1), 34-44.

Kaptelinin, V., \& Nardi, B. A. (2006). Acting with technology: Activity theory and interaction design. Mit Press.

Kaufmann, H., Schmalstieg, D., \& Wagner, M. (2000). Construct3D: a virtual reality application for mathematics and geometry education. Education and information technologies, 5(4), 263-276.

Krueger, M. W. (1983). Artificial reality.

Lackey, S. J., Salcedo, J. N., Szalma, J. L., \& Hancock, P. A. (2016). The stress and workload of virtual reality training: the effects of presence, immersion and flow. Ergonomics, 1-13.

Lanier, J. (1992). Virtual Reality: The Promise of the Future. Interactive Learning International, 8(4), 275-79.

Lanier, J., \& Biocca, F. (1992). An insider's view of the future of virtual reality. Journal of communication, 42(4), 150-172.

Lau, K. W., \& Lee, P. Y. (2015). The use of virtual reality for creating unusual environmental stimulation to motivate students to explore creative ideas. Interactive Learning Environments, 23(1), 3-18.

Levin, M. F., Weiss, P. L., \& Keshner, E. A. (2015). Emergence of virtual reality as a tool for upper limb rehabilitation: incorporation of motor control and motor learning principles. Physical therapy, 95(3), 415-425.

Lorenz, M., Busch, M., Rentzos, L., Tscheligi, M., Klimant, P., \& Frohlich, P. (2015, March). I'm There! The influence of virtual reality and mixed reality environments combined with two different navigation methods on presence. In Virtual Reality (VR), 2015 IEEE (pp. 223-224). IEEE.

Marti, S. (2006) Aspen Interactive Movie Map (1978). Recuperado en 2016-03-30 <https://www.youtube.com/watch?v=Hf6LkqgXPMU>

McLellan, H. (1996). Virtual realities. Handbook of research for educational communications and technology, 457-487. 
McLuhan, M. (1964). Understanding Media: The Extensions of Man. McGraw-Hill. Meshulam, L., Gauthier, J., Brody, C., Tank, D., \& Bialek, W. (2016). Can simple interactions capture complex features of neural activity underlying behavior in a virtual reality environment?. Bulletin of the American Physical Society.

Meshulam, L., Gauthier, J., Tank, D., \& Bialek, W. (2015, March). Interpreting collective neural activity underlying spatial navigation in virtual reality. In APS Meeting Abstracts (Vol. 1, p. 50009)

Milgram, P., \& Kishino, F. (1994). A taxonomy of mixed reality visual displays. IEICE TRANSACTIONS on Information and Systems, 77(12), 1321-1329.

Moreno Toledano, L. y Rogel Villalba, E. (2016). La simulación de la cultura a través de los mundos virtuales. El caso de Second Life: un acercamiento al potencial representacional, narrativo y retórico en las nuevas culturas mediáticas, Icono 14, volumen (14), pp. 1-25.

McLuhan, M., \& Fiore, Q. (1967). The medium is the message. New York, 123, 126-128 Naimark, M. (1978). Aspen Moviemap. Naimark.net. Recuperado en 2016-03-30 <http://www.naimark.net/projects/aspen.html>

Nogueira, P. A., Torres, V., \& Rodrigues, R. (2013). Automatic emotional reactions identification: a software tool for offline user experience research. In Entertainment computing-ICEC 2013 (pp. 164-167). Springer Berlin Heidelberg. Ong, S. K., \& Nee, A. Y. C. (2013). Virtual and augmented reality applications in manufacturing. Springer Science \& Business Media.

Ortega, M. D. R., Alvarado, L. 0., \& Higueruela, F. R. F. (2015). Advances in 3D Spatial Information Systems. Applications in cultural heritage and virtual archeology. Virtual Archaeology Review, 6(12), 77-91.

Ohta, Y., \& Tamura, H. (2014). Mixed reality: merging real and virtual worlds. Springer Publishing Company, Incorporated.

Otero Franco, A., Flores González, J. (2011). Realidad Virtual, un medio de comunicación de contenidos: Aplicación como herramienta educativa y factores de diseño e implantación en espacios públicos. Icono 14 (185-211).

Packer, R., \& Jordan, K. (2002). Multimedia: from Wagner to virtual reality. WW Norton \& Company.

Picard, R. W. (1995). Affective computing.

Psotka, J. (1995). Immersive training systems: Virtual reality and education and training. Instructional science, 23(5-6), 405-431. 
Reiners, D., Cruz-Neira, C., \& Neumann, C. (2015, March). Photorealistic 3D omnidirectional stereo simulator. In IS\&T/SPIE Electronic Imaging (pp. 939204939204). International Society for Optics and Photonics.

Rheingold, H. (1991). Virtual Reality: Exploring the Brave New Technologies. Simon \& Schuster Adult Publishing Group.

Riva, G., Mantovani, F., \& Gaggioli, A. (2004). Presence and rehabilitation: toward second-generation virtual reality applications in neuropsychology. Journal of neuroengineering and rehabilitation, 1(1), 1.

Rizzo, A. A., Schultheis, M., Kerns, K. A., \& Mateer, C. (2004). Analysis of assets for virtual reality applications in neuropsychology. Neuropsychological Rehabilitation, 14(1-2), 207-239.

Rubio-Tamayo, J. L. (2015). Creación Experimental en el Espacio Digital. Entornos Digitales Colaborativos y Laboratorios Multimedia.

Ruiz Martín, J.M. y Alcalá Mellado, J.R. (2016): Los cuatro ejes de la cultura participativa actual. De las plataformas virtuales al medialab, Icono 14, volumen (14), pp. 95-122. doi: 10.7195/ri14.v14i1.904

Ryan, M. L. (2001). Narrative as virtual reality: Immersion and interactivity in literature and electronic media. Johns Hopkins University Press.

Santo, A. (2016). La última frontera de la realidad virtual no es la tecnológica. FSGamer. Recuperado en 2016-03-10 de http://www.fsgamer.com/la-ultimafrontera-de-la-realidad-virtual.html

Sedig, K., \& Parsons, P. (2013). Interaction design for complex cognitive activities with visual representations: A pattern-based approach. AIS Transactions on Human-Computer Interaction, 5(2), 84-133.

Seidel, R. J., \& Chatelier, P. R. (Eds.). (2013). Virtual Reality, Training's Future?: Perspectives on Virtual Reality and Related Emerging Technologies (Vol. 6). Springer Science \& Business Media.

Sharar, S. R., Miller, W., Teeley, A., Soltani, M., Hoffman, H. G., Jensen, M. P., \& Patterson, D. R. (2014). Applications of virtual reality for pain management in burn-injured patients. Expert review of neurotherapeutics.

Sharp, H. (2003). Interaction design. John Wiley \& Sons.

Sherman, W. R., \& Craig, A. B. (2002). Understanding virtual reality: Interface, application, and design. Elsevier. 


\section{INNOVACIÓN TEÓRICA}

Shneiderman, B. (2016). Encounters with HCI Pioneers: A Personal Photo. A personal walk down the computer industry road. BY AN EYEWITNESS., 23, 54.

Smith-Atakan, S. (2006). Human-computer interaction. Cengage Learning EMEA

Stephenson, N. (1992). Snow Crash. Bantam Books

Steuer, J. (1992). Defining virtual reality: Dimensions determining telepresence. Journal of communication, 42(4), 73-93.

Sutherland, I. E. (1965). The ultimate display. Information Processing Techniques. Office, ARPA, OSD

Thorsteinsson, G. (2013). Developing an Understanding of the Pedagogy of Using a Virtual Reality Learning Environment (VRLE) to Support Innovation Education. The Routledge International Handbook of Innovation Education. Edited by LV Shavinina. Oxford: Routledge. ISBN-10, 415682215, 456-470.

Tu, H., Paolo, M., Vincent, C., Li, Y., \& Thimbleby, H. (2016). IWC Special Issue in Human Factors and Interaction Design for Critical Systems. Interacting with Computers, 28(2), 149-150.

Tudor, S., Carey, S., \& Dubey, R. (2015, July). Development and evaluation of a dynamic virtual reality driving simulator. In Proceedings of the 8th ACM International Conference on PErvasive Technologies Related to Assistive Environments (p. 55). ACM.

Una iniciativa virtual de Médicos sin Fronteras permite ponerse en la piel de los refugiados (2016, 9 de marzo). Infolibre [en línea]. Recuperado en 2016-03-30 <http://www.infolibre.es/noticias/mundo/2016/03/09/ una_iniciativa_virtual_medicos_sin_fronteras_permite_ponerse_piel_los_ refugiados_46178_1022.html >

Weinbaum (1935). Pygmalion's Spectacles.

Wexelblat, A. (Ed.). (2014). Virtual reality: applications and explorations. Academic Press. Wu, C. H., Huang, Y. M., \& Hwang, J. P. (2015). Review of affective computing in education/learning: Trends and challenges. British Journal of Educational Technology.

Zimmerman, J., Forlizzi, J., \& Evenson, S. (2007, April). Research through design as a method for interaction design research in HCI. In Proceedings of the SIGCHI conference on Human factors in computing systems (pp. 493-502). ACM. 